\title{
Disponibilidad y uso de TIC en escuelas latinoamericanas: incidencia en el rendimiento escolar
}

Marcela Román'

F. Javier Murillo"

\section{Resumen}

Enriquecer los ambientes de aprendizaje de los estudiantes mediante la incorporación de tecnologías de información y comunicación requiere que los centros educativos dispongan de computadoras y conectividad - en cantidad y calidad suficiente - para que docentes y estudiantes puedan incorporar dichas tecnologías en el proceso de enseñanza y aprendizaje. La investigación que se presenta estima la incidencia del acceso y uso de computadoras en el logro escolar que obtienen los estudiantes latinoamericanos de $6^{\circ}$ de primaria en Matemáticas y Lectura. Para ello, y mediante modelos multinivel de cuatro niveles (alumno, aula, escuela y país), se analiza información de dieciséis países de América Latina, cerca de 91.000 estudiantes de sexto grado y algo más de 3.000 docentes, disponible en la base de datos del Segundo Estudio Comparativo y Explicativo (SERCE) de la UNESCO. Con importantes variaciones entre países, los resultados dan cuenta de que apenas un tercio de los niños y niñas latinoamericanos que estudian $6^{\circ}$ grado dispone de una computadora en casa y más de la mitad de ellos afırma que nunca han utilizado una computadora en la escuela. Entre sus principales hallazgos se constata que un estudiante que cuente con una computadora en su hogar, que concurra a una escuela con más de diez computadoras, que los utilice al menos una vez por semana y tenga un profesor/a que use habitualmente la computadora en su casa, obtendrá un desempeño significativamente más alto en ambas áreas evaluadas: 23 puntos más en matemáticas y 25 en lectura.

\section{Palabras clave}

Acceso y Uso TIC - América Latina - Rendimiento escolar Educación primaria

I- Universidad Alberto Hurtado, Santiago,

Chile.

Contacto: mroman@cide.cl

Il-Universidad Autónoma de Madrid,

Madrid, España.

Contacto: javier.murillo@uam.es 


\title{
The use and availability of ICT in Latin American schools: the impact on school performance
}

Marcela Román'

F. Javier Murillo"

\begin{abstract}
Enriching learning environments for students by incorporating information and communication technologies requires that schools have both computers and connectivity - in sufficient quantity and quality - to be incorporated by teachers and students into the teaching and learning process. The following research estimates the impact of computer access and use on math and reading achievement among Latin American 6th grade students. For this purpose and through a four-level model (student, classroom, school, and country), we analyzed data from 16 countries in Latin America. The database corresponds to the Second Comparative and Explanatory Study (UNESCO), which gathered information on about 91,000 sixth graders and more than 3,000 teachers. With significant variations between countries, the results show that only a third of Latin American children studying in the 6th grade have a computer at home and more than half of them said they have never used a computer at school. One of the key findings is that a student who has a computer at home, attends a school with more than 10 computers, uses them at least once a week, and has a teacher who routinely uses a computer at home will achieve a significantly higher performance in both of the areas evaluated: 23 more points in math and 25 more in reading.
\end{abstract}

\section{Keywords}

ICT access and use - Latin America - Educational achievement Elementary education.

I- Universidad Alberto Hurtado, Santiago,

Chile.

Contact: mroman@cide.cl

II-Universidad Autónoma de Madrid,

Madrid, España.

Contact: javier.murillo@uam.es 


\section{Introducción}

Mientras América Latina se mantenga como la región de mayores desigualdades sociales y con sistemas educativos que no logran resolver los problemas de calidad y equidad de la educación ofrecida en ellos, seguirá siendo necesario profundizar en las condiciones y recursos con que cuentan y enfrentan sus poblaciones escolares en el proceso de aprender. Mirada que resulta indispensable no solo para hacer más comprensible los aprendizajes o desempeños que alcanzan los niños y jóvenes en los distintos países y contextos, sino que en tanto insumo relevante para la definición e implementación de políticas que contribuyan a incrementar la calidad educativa y su justa distribución.

Dotar de computadoras, software educativo y acceso a internet a las escuelas, ha sido una compartida preocupación y esfuerzo de los gobiernos latinoamericanos durante las últimas décadas. Ello bajo la premisa que tales tecnologías de información y comunicación (TIC) son quizás los recursos más relevantes en las dinámicas actuales de enseñar y aprender, no sólo por su indiscutida condición de principal puerta de acceso al conocimiento y a la información, sino porque con su irrupción ha cambiado la gramática del cómo enseñar y qué aprender en estas sociedades complejas, globalizadas e interconectadas (ENGEL, COLL; BUSTOS, 2010; PELGRUM; LAW, 2003; ROSEN; NELSON, 2008; KAZTMAN, 2010; SELWYN, 2011). Aparece así, del todo obvio y necesario que estos nuevos estudiantes: aprendices del nuevo milenio (PEDRÓ, 2006) o nativos digitales (BENNETT, MATON; KERVIN, 2008), puedan acceder a tales recursos e incorporarlos en su proceso de aprehender y construir la realidad, relacionarse y comunicarse con el mundo y los otros (COLL, 2004). En este desafío, la escuela se constituye en el principal espacio social y formativo que ha de permitir que esto ocurra para todos los niños, niñas y jóvenes, independientemente del país, contexto o condición socioeconómica familiar. En su interior, la exigencia se concretiza en los profesores y profesoras, para que ellos integren estos recursos como parte sustantiva de la dinámica y práctica cotidiana de enseñanza. En tal sentido, las TIC se incorporan al escenario educativo con la doble expectativa de apoyar la adquisición de habilidades y competencias propias de este siglo, al tiempo que aportar al mejoramiento de los desempeños de los estudiantes.

El estudio que se presenta, da cuenta de la disponibilidad de recursos TIC en las escuelas de 16 países de América Latina y profundiza en la relación entre acceso y uso de la computadora y el rendimiento escolar en Lectura y Matemáticas, en estudiantes latinoamericanos de educación primaria. Dicho rendimiento, corresponde al logro alcanzado por los alumnos, controlado por factores externos tales como el nivel socio-económico y cultural de las familias. Para ello, se utiliza la base de datos del Segundo Estudio Comparativo y Explicativo, SERCE, de la UNESCO, que evalúa el rendimiento de los estudiantes de $3^{\circ}$ y $6^{\circ}$ de primaria y estudia los factores asociados a tales desempeños. La información referida se analiza mediante Modelos Multinivel de cuatro niveles de análisis: alumno, aula, escuela y país.

En un primer apartado se revisa la literatura referida al acceso de TIC en las escuelas y sistemas educativos, así como de su aporte a los aprendizajes y desempeños escolares. El segundo capítulo comparte los propósitos y estrategia metodológica que sustenta el estudio, para posteriormente en un tercer punto, recorrer y describir los principales resultados de la investigación. Al finalizar, se discuten los resultados con la evidencia disponible para desde allí, ofrecer algunas conclusiones y recomendaciones al respecto.

\section{Revisión de la literatura}

Diferentes estudios e investigaciones internacionales son concluyentes respecto de las importantes brechas de acceso a TIC entre los estudiantes en función del nivel de 
desarrollo del país, tanto a nivel de hogares como en las escuelas. En efecto, según recientes datos de la OCDE - Organización para la Cooperación y Desarrollo Económicos (2010), todas las escuelas de los países que conforman esta organización, con la excepción de México, disponen de computadoras, y cerca de un 90 por ciento de ellas cuenta además con conectividad a internet. Sin embargo, otro es el panorama a nivel regional, donde más de la mitad (54\%) de las escuelas latinoamericanas no cuenta con computadoras para uso de los estudiantes (FALUS; GOLBERG, 2010). Promedio que varía importantemente según el país en cuestión. En el extremo superior y, con casi cobertura universal de TIC en su sistema escolar, se ubican Chile y Cuba, mientras que en el otro extremo, más del 85 por ciento de las escuelas de Guatemala, Nicaragua, Paraguay y República Dominicana, no disponen de computadoras.

Importantes son también las diferencias respecto de la tasa estudiantes/computadora. Así, mientras que en las escuelas de los países OCDE, este indicador es de 5:1 en las escuelas (OCDE, 2010), en América Latina, la mejor cifra es la de Uruguay con una relación de 27:1. Respecto de la conectividad, las cifras son significativamente menores y las brechas entre países mayores. El 60 por ciento de las escuelas mexicanas no disponen de internet, cifra que alcanza el 56 por ciento en Brasil, el 42 por ciento para Argentina y el 32 por ciento en Uruguay. Chile muestra las mejores cifras, con un 92 por ciento de sus escuelas con conectividad.

Por otra parte, la información constata que son los estudiantes con mayores recursos quienes tienen un mayor acceso a la computadora en la escuela y que esta disponibilidad es también mayor entre quienes asisten a escuelas privadas. Respecto de la ubicación, las escuelas rurales siguen estando muy rezagadas respecto de las urbanas, mientras que la conectividad (internet), es muy inferior en relación con la disponibilidad de computadoras en todas las escuelas y contextos geográficos (SUNKEL,
TRUCCO; MÖLLER, 2011; ROMÁN; MURILLO, 2012). Pero, si las cifras de acceso a TIC en las escuelas de la región ya muestran tales desigualdades, los datos a nivel de los hogares latinoamericanos son aún más lapidarios: los mayores porcentajes apenas hablan de un 30\% de estudiantes con acceso a una computadora en casa (Chile), mientras que en el otro extremo, no supera el 5 por ciento en países como El Salvador (SUNKEL, 2007).

\section{El aporte de las TIC a los aprendizajes y desempeños escolares}

A pesar de la ingente literatura sobre la aportación de las TIC en el rendimiento académico de los estudiantes, tras décadas de esfuerzos por equipar y dotar de recursos tecnológicos a los sistemas escolares y enriquecer los ambientes de aprendizajes en las escuelas, la principal certeza de que disponemos es que para alcanzar mejores aprendizajes no basta ni es suficiente con poner a disposición de alumnos y docentes tales tecnologías (KOZMA, 2005; CARSTENS; PELGRUM, 2009; CHONG, 2011).

De manera concreta, la revisión y sistematización de diversos estudios y experiencias que han abordado la relación entre TIC y rendimientos escolares, no permiten extraer conclusiones claras y definitorias, como queda reflejado en los meta-análisis de Kulik (1994, 2003), Eng (2005), Condie, Munro, Seagraves y Kenesson (2007) y de Dynarski y sus colaboradores (2007). Ellos coinciden en señalar que una de las principales complicaciones para comparar y encontrar regularidades entre unos y otros estudios, radica en la diferente forma de definir efecto escolar, así como en la metodología para medirlo. No obstante ello, en la mayoría de los estudios de las últimas décadas que fueron analizados, se encuentra un efecto positivo aunque moderado de las TIC en el aprendizaje y especialmente referido a las habilidades de escritura en primaria (KULIK, 2003; ENG, 2005). Desde su mirada, estos hallazgos pueden estar reflejando dos aspectos 
relevantes en el tiempo. Por una parte, el mayor desarrollo técnico y metodológico para medir y controlar tales efectos, en estas últimas décadas y, por otra, una mayor limitación de las posibilidades educativas de los recursos y herramientas tecnológicas disponibles en las décadas anteriores.

El estudio de la OCDE (2010), a partir de datos de PISA - Program for International Student Assessment 2006 y controlando por nivel económico, social y cultural, encuentra mayor efecto en el rendimiento de Ciencias en función del tiempo de uso: treinta puntos porcentuales de ventaja para aquellos estudiantes que han usado una computadora entre uno a tres años; 51 puntos para los que lo han usado de tres a cinco años y 61 puntos para los que lo han usado durante más de cinco años.

Es importante señalar que la revisión realizada da cuenta de un importante grupo de estudios que encuentran efectos muy limitados o simplemente nulos al analizar la relación entre TIC y logro escolar a nivel internacional (ANGRIST; LAVY, 2002; GOOLSBEE; GURYAN, 2006; ROUSE y KRUEGER, 2004).

La investigación a nivel regional, sin duda mucho menor, tampoco muestra grandes consensos ni es concluyente. Así por ejemplo, las pocas evaluaciones de las iniciativas 1 a 1 (una computadora por estudiante) en la región, tampoco llegan a consensos respecto de su aporte al rendimiento escolar (VALIENTE, 2010). En el caso de Ecuador, se constata un efecto significativo en matemáticas, pero no en lenguaje (CARRILLO; ONOFA; PONCE, 2010), mientras que la experiencia en escuelas rurales peruanas, no encuentra evidencia de efectos en los aprendizajes (SANTIAGO et. al., 2010).

La Investigación Iberoamericana sobre Eficacia Escolar (MURILLO, 2007), muestra que la calidad y adecuación de los recursos TIC aparecen relacionados con el rendimiento en Lengua en $3^{\circ}$ de Primaria. Hallazgos que son ratificados mediante una explotación especial de estos datos (ROMÁN, 2008), donde se constata que los alumnos cuyos docentes usan recursos
TIC en las actividades destinadas al aprendizaje, logran rendimientos significativamente mayores tanto en lengua como en matemáticas. Por último y respecto de uso y aprendizaje, aparecen como relevantes i) la frecuencia y tiempo de uso de TIC por parte del estudiante; ii) desde los docentes: el nivel de dominio de estos recursos; la valoración de las TIC como herramientas didácticas; la confianza y seguridad en el uso; tiempo para la planificación de la enseñanza, y iii) ciertas condiciones institucionales y apoyos desde la dirección de las escuelas y centros, entre otros (VENEZKY, 2002; KULICK, 2003; PELGRUM; LAW, 2003; SELWYN, 2004; WEB; COX, 2004; TRUCANO, 2005; DYNARSKI et. al., 2007; LAW et. al., 2008; ROMÁN, 2010). En todo caso, lo que si se observa es que cuando las TIC forman parte de la experiencia cotidiana del aula, hay mayores evidencias de su impacto en el aprendizaje y el desempeño de los estudiantes (CONDIE et. al., 2007; CLARO, 2010).

\section{La investigación: objetivos y estrategia metodológica}

La investigación se plantea como objetivos:

1. Estimar y caracterizar el acceso y uso de computadoras para estudiantes y docentes, en escuelas primarias de América Latina.

2. Determinar la incidencia del acceso y tipo de uso de computadoras por parte de los estudiantes y docentes latinoamericanos de $6^{\circ}$ de Primaria, en el logro escolar que tales estudiantes alcanzan en las áreas de Matemáticas y Lectura.

Se entiende por logro escolar el desempeño de los estudiantes controlado por factores externos tales como el nivel socioeconómico y cultural de las familias; es decir, partiendo de un planteamiento de valor agregado.

Para ello, se utiliza la base de datos del Segundo Estudio Comparativo y Explicativo, SERCE, de la UNESCO (2008), que evalúa el rendimiento de los estudiantes de $3^{\circ}$ y $6^{\circ}$ de primaria en Lectura y Matemáticas, y estudia 
los factores asociados a tales desempeños. Para los objetivos de este estudio se trabaja sólo con datos de $6^{\circ}$ grado que se analizan bajo el enfoque de Modelos Multinivel de cuatro niveles de análisis: alumno, aula, escuela $\mathrm{y}$ país, para alcanzar el segundo objetivo.

\section{Muestra}

El estudio considera datos de dieciséis países, 2.969 escuelas, 3.903 docentes, y 91.223 estudiantes de sexto grado de Primaria (cuadro 1). Esta muestra, estadísticamente representativa para cada país, fue seleccionada mediante muestreo aleatorio estratificado de conglomerados. Los criterios para la estratificación fueron el tipo de administración (privada-pública), contexto de la escuela (rural- urbano) y tamaño. A partir de cada escuela seleccionada, se eligen todos los estudiantes y docentes del curso analizado (en este caso, de $6^{\circ}$ primaria).

Cuadro 1. Muestra del estudio: número de escuelas, docentes y estudiantes

\begin{tabular}{|c|c|c|c|}
\hline País & Escuelas & Docentes & Estudiantes \\
\hline Argentina & 167 & 353 & 6.696 \\
\hline Brasil & 157 & 245 & 5.456 \\
\hline Colombia & 203 & 207 & 6.035 \\
\hline Costa Rica & 171 & 150 & 4.766 \\
\hline Cuba & 206 & 383 & 5.910 \\
\hline Chile & 165 & 263 & 7.025 \\
\hline Ecuador & 192 & 215 & 5.427 \\
\hline El Salvador & 182 & 235 & 6.346 \\
\hline Guatemala & 231 & 267 & 5.560 \\
\hline México & 160 & 220 & 4.861 \\
\hline Nicaragua & 205 & 250 & 6.789 \\
\hline Panamá & 155 & 247 & 5.655 \\
\hline Paraguay & 209 & 208 & 4.839 \\
\hline Perú & 165 & 243 & 4.701 \\
\hline R. Dominicana & 183 & 114 & 4.646 \\
\hline Uruguay & 218 & 303 & 6.511 \\
\hline Total & 2.969 & 3.903 & 91.223 \\
\hline
\end{tabular}

Fuente: Elaboración propia a partir de los datos del SERCE.

Las variables utilizadas son de tres tipos: de producto, de ajuste e independientes o explicativas.
- Las variables de producto son el Rendimiento en Matemáticas y en Lectura de los estudiantes de sexto grado de Primaria. Ambas están estimadas mediante Teoría de Respuesta al Ítem y escaladas con una media de quinientos y una desviación típica de cincuenta.

- Las variables de ajuste son cinco:

1. Nivel socioeconómico de la familia del estudiante, variable tipificada y obtenida a partir de la profesión de los padres y de las posesiones familiares;

2. Nivel cultural de la familia del estudiante, obtenida como promedio de la titulación máxima conseguida por ambos padres, tipificada;

3. Género, variable dummy $y^{1}$; toma el valor 1 si la persona es mujer y cero en cualquier otro caso

4. Lengua materna, si la lengua materna del estudiante es el español/portugués ${ }^{2}$ u otra; variable dummy;

5. Años de preescolarización del estudiante, número de años que asistió a algún centro educativo o asistencial antes de la educación obligatoria.

- Las variables independientes o explicativas utilizadas son:

1. Acceso de computadoras en el hogar estudiantes (dummy),

2. Frecuencia de uso de la computadora en la escuela estudiante (ordinal cinco alternativas),

3. Uso habitual de la computadora por el docente en casa (dummy), o en la escuela (dummy),

1- Las variables dummy, también conocidas como variables binarias 0 dicotómicas, solo pueden asumir los valores 0 y 1 , indicando respectivamente ausencia o presencia de una cualidad 0 atributo.

2- Las pruebas SERCE de matemática y lectura para ambos grados evaluados, cuentan con versiones en español y portugués (usadas exclusivamente en la medición en Brasil). Por tal razón, la variable "lengua materna", se considera el español u otra cualquiera en la totalidad de los países participantes del SERCE, excepto Brasil, en donde dicha variable puede tomar también dos valores, según se trate del portugués 0 de cualquiera otra distinta del portugués. 
4. Número de computadoras en la escuela (dummy: diez o menos computadoras, más de diez).

- Las variables fueron recogidas mediante los siguientes instrumentos:

1. Variables de producto (Rendimiento en Matemáticas y en Lectura): recogidas mediante pruebas estandarizadas validadas para todos los países. Las pruebas consideran dos dimensiones: los elementos curriculares comunes en la región y el enfoque de habilidades para la vida.

2. Variables de ajuste: obtenidas mediante cuestionarios dirigidos a estudiantes (género y lengua materna) sus familias (nivel cultural y situación socio-económica de la familia y años de preescolarización del estudiante).

3.Variables explicativas o independientes: obtenidas de diferentes cuestionarios: dirigidas a los estudiantes, docentes y dirección de la escuela.

Se utilizaron Modelos Multinivel de cuatro niveles de análisis. Concretamente se desarrollaron dos procesos de Modelamiento multinivel, uno de cada una de las variables de producto. El procedimiento fue el siguiente: a) estimar el modelo nulo; b) calcular el modelo con las variables de ajuste; y c) incluir en el modelo ajustado cada las variables de acceso y uso de TIC y estimar la aportación de las mismas.

De esta forma se estimaron dos modelos multinivel análogos al siguiente:

$Y_{i j k l}=\beta_{0 j k l}+\beta_{1 j k l} N S E_{i j k l}+\beta_{2 j k l} N C u l t_{i j k l}+\beta_{3 j k l} \operatorname{Preesc}_{i j k l}+$ $\beta_{4 j k l}$ Genero $_{i j k l}+\quad \beta_{5 j k l} L M_{i j k l}+\beta_{6 j k l}$ Acce_Compu ${ }_{i j k l}+$ $\beta_{7 j k l}$ Fresc_uso_esc $c_{i j k l}+\beta_{8 k l} U_{s o} \quad$ prof_casa ${ }_{j k l}+$ $\beta_{9 k l} U s o \_p r o f \_e s c_{j k l}+\beta_{10 l}$ Compus_esc $c_{k l}+\varepsilon_{i j k l}$

$\beta_{0 j k l}=\beta_{0}+\varphi_{0 l}+v_{0 k l}+\mu_{0 j k l}$

$\beta_{1 j k l}=\beta_{1}+\varphi_{1 l}+v_{1 k l}+\mu_{1 j k l} \ldots \beta_{7 j k l}=\beta_{7}+\varphi_{7 l}+v_{7 k l}+\mu_{7 j k l}$ $\beta_{8 k l}=\beta_{8}+\varphi_{8 l}+v_{8 k l} \beta_{9 j k l}=\beta_{9}+\varphi_{9 l}+v_{9 k l}$ $\beta_{10 l}=\beta_{11}+\varphi_{10 l}$
Con:

$$
\begin{aligned}
& {\left[\varepsilon_{0 \mathrm{ijk}}\right] \sim N\left(0, \Omega_{\varepsilon}\right): \Omega_{\varepsilon}=\left[\sigma^{2}\right]} \\
& {\left[\mu_{0 \mathrm{jk}}\right] \sim N\left(0, \Omega_{\mu}\right): \Omega_{\mu}=\left[\sigma^{2}{ }_{\mu 0}\right]} \\
& {\left[v_{0 \mathrm{k}}\right] \sim N\left(0, \Omega_{v}\right): \Omega_{\mu}=\left[\sigma_{\mu 0}^{2}\right]} \\
& {\left[\varphi_{01}\right] \sim N\left(0, \Omega_{\varphi}\right): \Omega_{\varphi}=\left[\sigma_{\varphi 0}^{2}\right]}
\end{aligned}
$$

Donde, para cada $i=$ estudiante, $j=$ aula, $K=$ escuela y $l=$ país:

$Y_{i j k l}$, Son las diferentes medidas de desempeño del estudiante,

$N S E_{i j k}$, Nivel socioeconómico de la familia del estudiante,

NCult $_{i j k l}$, nivel cultural de la familia del estudiante, Preescol $_{i j k l}$, años de preescolarización del estudiante, Genero $_{i j k k}$, si el estudiante es niño o niña,

$L M_{i j k l}$, lengua materna del estudiante: español/ portugués u otra,

Acce_compu $u_{i j k l}$, si el estudiante tiene acceso a computadora en casa o no,

Frec_uso_esc $c_{i j k l}$, frecuencia del uso de la computadora en la escuela por el estudiante,

Uso_prof_casa $a_{j k}$, si el docente usa habitualmente la computadora en casa,

Uso_prof_esc ${ }_{\mathrm{jk}}$, si el docente usa habitualmente la computadora en la escuela,

Compues_esc $c_{\mathrm{kl}}$, si la escuela tiene 10 computadoras o menos; o más.

\section{Resultados}

En coherencia con los objetivos del estudios, los resultados se presentan en dos grandes apartados: en primer lugar se describe y caracteriza la situación de acceso y uso de las computadoras en Educación primaria en América Latina, referida a estudiantes, docentes y escuelas, y, en segundo lugar, se estudia su incidencia en el logro escolar en Matemáticas y Lectura.

\section{Disponibilidad y uso de recursos TIC:}

Disponibilidad Computadora en hogares estudiantes

Apenas un tercio de los niños y niñas latinoamericanos que estudian $6^{\circ}$ grado dispone de un computadora en casa $(31,8 \%)$ 
(Cuadro 2). Es posible agrupar a los países en cuatro grandes grupos: a) Países en donde cerca de la mitad de los estudiantes cuentan con computador en sus casas (Chile y Argentina); b) Países en los cuales el porcentaje de estudiantes que cuenta con un computador en sus hogares se mueve entre un 35 por ciento y un 46 por ciento (Costa Rica, México, Rep. Dominicana, Uruguay, Panamá, Ecuador y Brasil; c) Países con porcentajes que van entre un 25 por ciento y un 30 por ciento (Colombia, El Salvador, Perú, Paraguay y Guatemala y, d) Países con menos de un 20 por cineto de los estudiantes con acceso a una computadora en sus hogares (Nicaragua y Cuba).

Cuadro 2. Porcentaje de estudiantes de $6^{\circ}$ grado con computadora en casa, por país

\begin{tabular}{l:c} 
& Total \\
\hline Argentina & 49,62 \\
Brasil & 36,17 \\
Colombia & 29,11 \\
Costa Rica & 46,41 \\
Cuba & 8,91 \\
Chile & 50,39 \\
Ecuador & 37,36 \\
El Salvador & 27,83 \\
Guatemala & 25,98 \\
México & 43,20 \\
Nicaragua & 17,41 \\
Panamá & 40,37 \\
Paraguay & 26,74 \\
Perú & 27,45 \\
Rep. Dominicana & 42,39 \\
Uruguay & 40,70 \\
\hline Promedio Países & 34,38 \\
Total AL & 31,76 \\
\hline Pro &
\end{tabular}

Fuente: Elaboración propia.

El 71,4 por ciento de los estudiantes que asisten a centros privados urbanos tiene acceso a computadora en casa, cifra que solo llega a un 29,6 por ciento para los estudiantes de centros urbanos públicos y a un 12,0 por cineto para los estudiantes de zonas rurales (gráfico 1). Esta importante situación de desigualdad es general en todos los países, aunque es más aguda en Brasil y Perú. Mientras que el 80 por ciento de los niños y niñas que en Brasil asisten a centros privados tiene computadora en casa, solo lo tiene el 6,6 por ciento de los que viven en zonas rurales. En Perú, esta diferencia es de 64,9 por ciento a 6,35 por ciento.

Gráfico 1. Porcentaje de estudiantes de $6^{\circ}$ grado con computadora en casa, por país

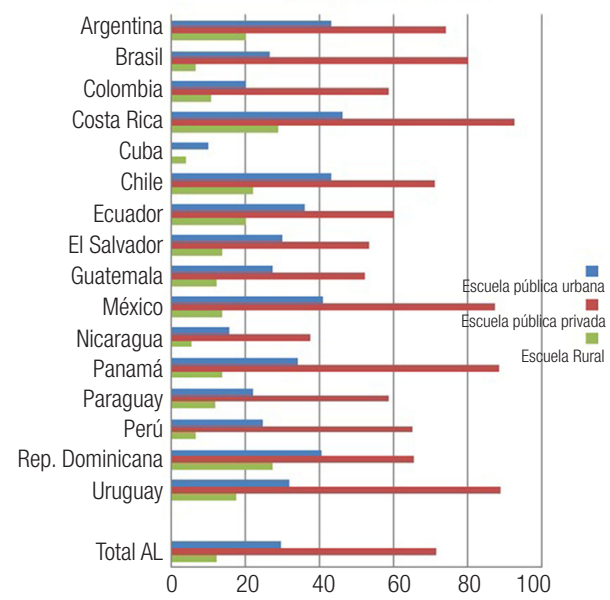

Fuente: Elaboración propia.

\section{Uso de la computadora en la escuela y hogares}

Por otra parte, algo más de la mitad de los estudiantes latinoamericanos afirma que nunca utilizan al computadora en la escuela $(51,8 \%)$. Estas cifras superan el 70 por ciento en Guatemala y Paraguay, mientras que está en torno al 2 por ciento en Cuba (cuadro 3). Respecto de la frecuencia, sólo un 31,2 por ciento señala usarlo de forma habitual. Así, son cuatro los países en donde más de la mitad de los estudiantes de $6^{\circ}$ señalan usar la computadora en la escuela una o más veces por semana: Cuba (85,7\%), Ecuador (58,2\%), Costa Rica $(52,4 \%)$ y Colombia $(51,5 \%)$. En el otro extremo, Paraguay $(18,0 \%)$ y Brasil $(22,1 \%)$. 
Cuadro 3. Frecuencia de uso de la computadora en la escuela según los estudiantes de sexto curso de Primaria de América Latina (sobre quienes dicen usar PC en la escuela)

\begin{tabular}{|c|c|c|c|c|}
\hline & $\begin{array}{l}\text { Una o más veces por } \\
\text { semana }\end{array}$ & $\begin{array}{c}\text { Una o más veces por } \\
\text { mes }\end{array}$ & $\begin{array}{c}\text { Cada dos o tres } \\
\text { meses }\end{array}$ & Nunca \\
\hline Argentina & 29,78 & 10,33 & 4,14 & 55,75 \\
\hline Brasil & 22,12 & 10,45 & 8,50 & 58,93 \\
\hline Colombia & 51,46 & 14,42 & 5,41 & 28,72 \\
\hline Costa Rica & 52,43 & 12,93 & 5,67 & 28,97 \\
\hline Cuba & 85,67 & 8,68 & 3,52 & 2,12 \\
\hline Chile & 42,27 & 18,45 & 12,17 & 27,11 \\
\hline Ecuador & 58,22 & 12,17 & 5,59 & 24,03 \\
\hline El Salvador & 38,46 & 10,86 & 5,36 & 45,32 \\
\hline Guatemala & 17,33 & 4,50 & 2,31 & 75,85 \\
\hline México & 43,04 & 11,12 & 5,48 & 40,36 \\
\hline Nicaragua & 18,15 & 6,25 & 6,10 & 69,50 \\
\hline Panamá & 48,40 & 11,27 & 4,47 & 35,86 \\
\hline Paraguay & 18,01 & 6,61 & 4,27 & 71,11 \\
\hline Perú & 37,39 & 15,88 & 8,09 & 38,64 \\
\hline Rep. Dominicana & 30,60 & 8,66 & 5,56 & 55,18 \\
\hline Uruguay & 34,03 & 10,23 & 5,87 & 49,88 \\
\hline Promedio Países & 39,21 & 10,80 & 5,78 & 44,21 \\
\hline Total AL & 31,20 & 10,58 & 6,46 & 51,76 \\
\hline
\end{tabular}

Fuente: Elaboración propia

Por su parte, prácticamente la mitad de los docentes dice usar la computadora en su casa $(48,2 \%)$, mientras que sólo un 36,12 por ciento señala hacerlo en la escuela, con importantes diferencias entre países (cuadro 4). Más del 70 por ciento de los docentes salvadoreños y paraguayos no usan computadora en ningún espacio, cifra que es de apenas el 0,8 por ciento en Cuba o el 6,4 por ciento en Costa Rica. Respecto del uso de la computadora en la escuela, los datos hablan también de importantes diferencias: mientras que en algunos países es excepcional, en otros es una práctica muy extendida, como en Cuba donde el 82 por ciento de los docentes afirma hacerlo.

Cuadro 4. Porcentaje de docentes que imparten $6^{\circ}$ grado que usan la computadora todas las semanas en distintos lugares, por país

\begin{tabular}{|c|c|c|c|}
\hline & No usa & Hogar & Escuela \\
\hline Argentina & 22,30 & 62,59 & 17,03 \\
\hline Brasil $^{\star}$ & - & - & - \\
\hline Colombia & 18,99 & 57,83 & 30,38 \\
\hline Costa Rica & 9,40 & 39,85 & 10,90 \\
\hline Cuba & 0,76 & 1,78 & 81,93 \\
\hline Chile & 6,45 & 66,31 & 43,82 \\
\hline Ecuador & 33,48 & 33,48 & 18,34 \\
\hline El Salvador & 72,97 & 36,09 & 31,94 \\
\hline Guatemala & 38,62 & 44,31 & 6,91 \\
\hline México & 28,44 & 40,83 & 47,25 \\
\hline Nicaragua & 66,00 & 4,40 & 8,80 \\
\hline Panamá & 38,95 & 21,40 & 20,70 \\
\hline Paraguay & 72,30 & 5,76 & 3,96 \\
\hline Perú & 19,92 & 42,54 & 23,13 \\
\hline Rep. Dominicana & 39,43 & 29,14 & 2,29 \\
\hline Uruguay & 17,16 & 64,36 & 30,03 \\
\hline Promedio Países & 32,35 & 36,71 & 25,16 \\
\hline Total AL & 29,93 & 48,21 & 36,19 \\
\hline
\end{tabular}

Nota: $\left(^{*}\right)$ En Brasil no se aplicó esa pregunta a los docentes.

Fuente: Elaboración propia 
Tipo y frecuencia de uso de la computadora

Cuadro 5. Distribución de los docentes de $6^{\circ}$ grado en función de la frecuencia de uso de la computadora para diferentes fines, total América Latina

\begin{tabular}{l|c:c:c}
\hline & Frecuente & Ocasional & Nunca \\
\hline Comunicarse & 14,09 & 25,02 & 60,89 \\
Buscar Información & 37,78 & 20,91 & 41,31 \\
Procesador de Textos & 39,45 & 19,15 & 41,40 \\
Hoja de Cálculo & 14,82 & 26,15 & 59,02 \\
Presentaciones & 17,29 & 29,02 & 53,69 \\
Otros fines & 20,41 & 21,10 & 58,49 \\
\hline
\end{tabular}

Fuente: Elaboración propia

Los datos indican que el principal uso que los docentes dan a los computadores es como procesador de texto. Un 41,1 por ciento de los docentes a nivel regional, dicen que lo usan con frecuencia con dicho fin. La búsqueda de información aparece en segundo lugar (34,8\%), seguido del uso dado para hacer presentaciones (21,6\%). Sólo un 19,0 por ciento lo utiliza frecuentemente como hoja de cálculo y un 14,1 por ciento para comunicarse (cuadro 5).

Un estudio detallado por países muestra interesantes perfiles (gráfico 2):

- Cuba: país con el mayor porcentaje de docentes que usan computadora en la escuela; este uso es especialmente como Hoja de Cálculo y para presentaciones audiovisuales, pero no lo usan ni para comunicarse ni para buscar información.

- México: país en donde cerca de la mitad de sus docentes señalan usar computadora en la escuela; los principales usos dados son como procesador de textos (35\%) y para presentaciones (32\%).

- Chile: Los docentes de este país declaran un uso especialmente importante para comunicarse (usar correo o chat, 41\%) y buscar información (72\%).
- Brasil: Los docentes declaran un uso mayoritario como procesador de textos (73\%) y para buscar información (72\%)

- Paraguay: país con el menor porcentaje de docentes que usan la computadora, es también el país que muestra los menores porcentajes de uso en cualquiera de los fines analizados.

\section{Disponibilidad de computadoras en las escuelas latinoamericanas}

Los datos señalan que el 82,4 por ciento de las escuelas latinoamericanas cuenta con al menos una computadora. En Brasil, Chile y Cuba todos los centros educativos de primaria cuentan con al menos una computadora, mientras que en Guatemala esta cifra es sólo de un 16 por ciento, en República Dominicana de un 29,6 por ciento y en Paraguay de un 33 por ciento (cuadro 6).

El 28 por ciento de las escuelas de América Latina no tiene ninguna computadora con conexión a Internet; el 23,2 por ciento no dispone de ninguna computadora para uso de los estudiantes de sexto grado y un 23,5 por ciento no cuenta con este recurso para uso de los docentes.

Considerando las escuelas que tienen alguna computadora, se observa que el número promedio de computadoras por escuela es muy bajo. Así, para el conjunto de la región el número medio de computadoras en cada escuela es de 9,5. Cifra que en sus extremos es de 3,5 en Cuba y de 16,6 en República Dominicana. Es interesante observar que existe una fuerte relación entre el porcentaje de escuelas sin ninguna computadora y el número de computadoras promedio en las escuelas que tienen $(r=0,61)$, lo que indica, en cierta medida, el énfasis en la distribución de recursos educativos: o pocas para muchas o muchas para pocas. 
Gráfico 2. Distribución de los docentes de $6^{\circ}$ grado en función de la frecuencia de uso de la computadora para diferentes fines, por país

\section{COMUNICARSE}
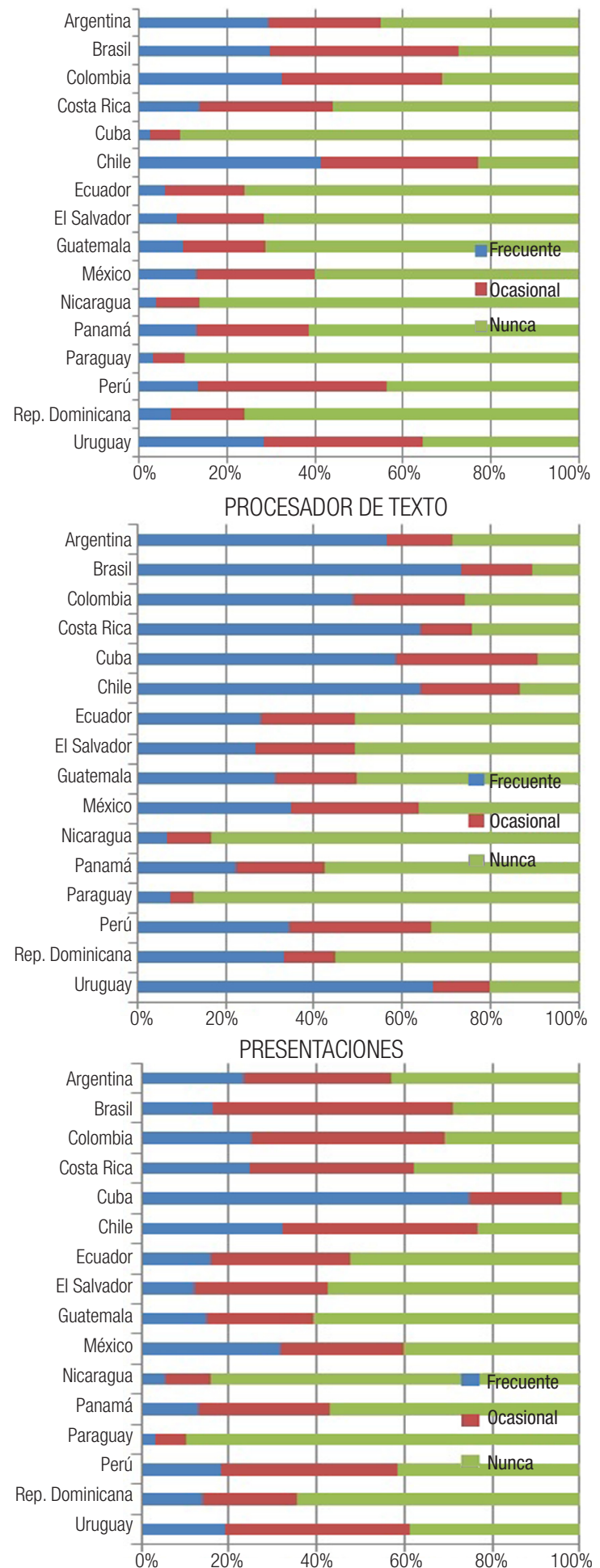

Fuente: Elaboración propia

\section{BUSCAR INFORMACIÓN}
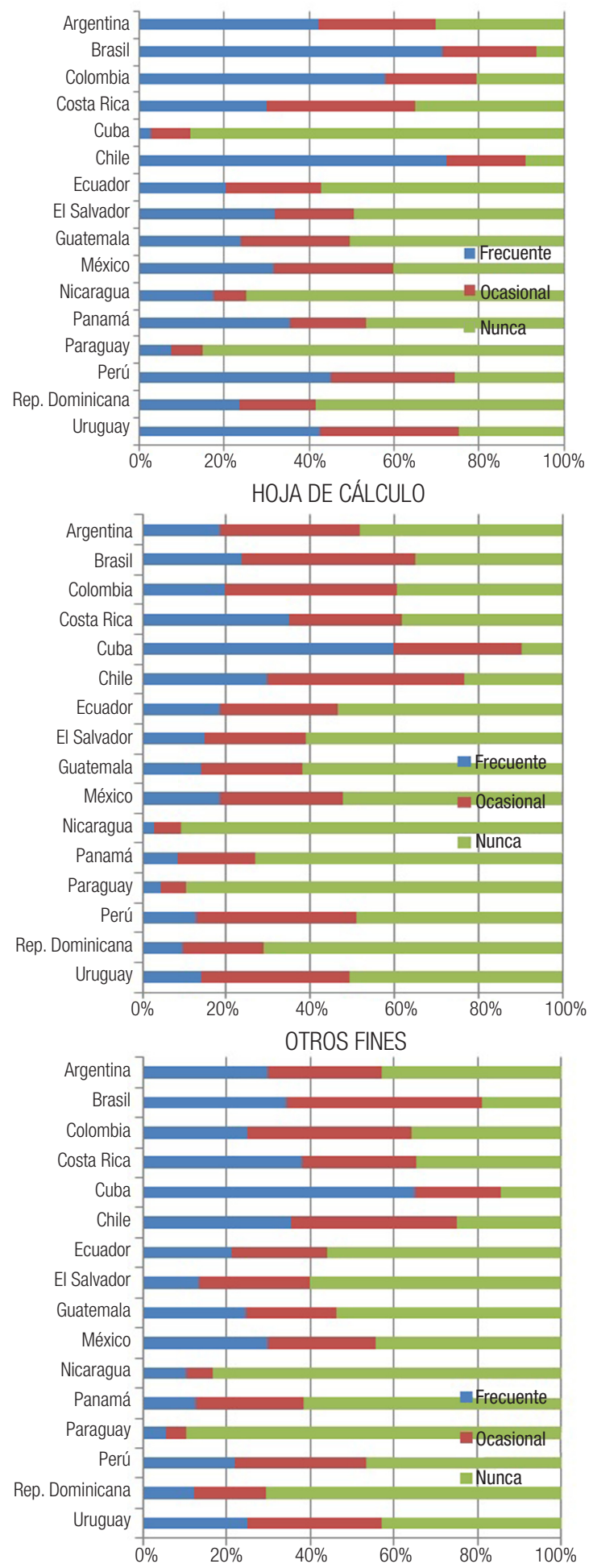
Cuadro 6. Porcentaje de escuelas de América Latina sin computadora y numero medio de computadoras entre las escuelas que tienen alguna. Total (con conexión a Internet) por país

\begin{tabular}{|c|c|c|c|c|}
\hline \multicolumn{3}{|c|}{ Media de computadoras por escuela } & \multicolumn{2}{|c|}{ Con conexión a Internet } \\
\hline & $\begin{array}{l}\% \text { escuelas sin } \\
\text { computadora }\end{array}$ & $\begin{array}{l}\text { Media de computadoras } \\
\text { por escuela }\end{array}$ & $\begin{array}{l}\% \text { escuelas sin } \\
\text { computadora }\end{array}$ & $\begin{array}{l}\text { Media de computadoras } \\
\text { por escuela }\end{array}$ \\
\hline Argentina & 15,40 & 7,91 & 67,47 & 7,20 \\
\hline Brasil & 0,00 & 10,26 & 0,00 & 12,06 \\
\hline Colombia & 20,24 & 15,48 & 42,61 & 13,83 \\
\hline Costa Rica & 34,64 & 10,84 & 68,72 & 13,62 \\
\hline Cuba & 0,00 & 3,44 & 98,03 & 3,01 \\
\hline Chile & 0,00 & 16,81 & 9,23 & 17,49 \\
\hline Ecuador & 16,53 & 7,21 & 39,82 & 9,05 \\
\hline El Salvador & 37,98 & 10,11 & 65,63 & 16,54 \\
\hline Guatemala & 84,33 & 10,78 & 95,05 & 14,29 \\
\hline México & 14,07 & 7,42 & 18,02 & 7,07 \\
\hline Nicaragua & 36,52 & 12,90 & 38,83 & 8,86 \\
\hline Panamá & 20,57 & 13,59 & 23,63 & 17,03 \\
\hline Paraguay & 67,39 & 8,47 & 78,05 & 7,46 \\
\hline Perú & 39,23 & 10,17 & 45,90 & 15,30 \\
\hline Rep. Dominicana & 70,45 & 14,89 & 81,06 & 7,74 \\
\hline Uruguay & 8,41 & 6,07 & 53,27 & 5,49 \\
\hline Promedio Países & 29,11 & 10,40 & 51,58 & 11,00 \\
\hline Total AL & 17,56 & 10,02 & 28,13 & 11,68 \\
\hline
\end{tabular}

Fuente: Elaboración propia

Cuadro 7. Porcentaje de escuelas de América Latina sin computadora y número medio de computadoras entre las escuelas que tienen alguna para uso de los estudiantes de $6^{\circ}$, para uso de los docentes y para uso del personal administrativo, por país.

\begin{tabular}{|c|c|c|c|c|c|c|}
\hline & \multicolumn{2}{|c|}{ Para uso de los estudiantes de $6^{\circ}$} & \multicolumn{2}{|c|}{ Para uso de los docentes } & \multicolumn{2}{|c|}{$\begin{array}{l}\text { Para uso del personal } \\
\text { administrativo }\end{array}$} \\
\hline & $\% \sin$ & Media & $\% \sin$ & Media & $\% \sin$ & Media \\
\hline Argentina & 52,25 & 9,80 & 44,43 & 4,23 & 28,79 & 1,63 \\
\hline Brasil &, 00 & 10,78 &, 00 & 4,46 &, 00 & 2,73 \\
\hline Colombia & 26,95 & 12,04 & 30,39 & 4,13 & 25,16 & 2,63 \\
\hline Costa Rica & 60,47 & 14,79 & 53,65 & 4,32 & 46,68 & 2,39 \\
\hline Cuba & 8,41 & 3,54 & 4,45 & 3,38 & 17,94 & 3,18 \\
\hline Chile & 2,88 & 12,17 & 6,10 & 5,62 & 7,17 & 3,64 \\
\hline Ecuador & 28,13 & 9,33 & 29,81 & 3,96 & 30,77 & 2,04 \\
\hline El Salvador & 57,02 & 14,92 & 44,72 & 5,54 & 51,43 & 2,30 \\
\hline Guatemala & 91,95 & 17,27 & 89,61 & 2,94 & 87,85 & 1,64 \\
\hline México & 18,82 & 6,44 & 21,59 & 4,65 & 27,71 & 2,17 \\
\hline Nicaragua & 37,66 & 15,23 & 37,58 & 4,72 & 35,75 & 1,84 \\
\hline Panamá & 21,55 & 14,10 & 21,50 & 5,29 & 20,63 & 2,89 \\
\hline Paraguay & 75,41 & 12,33 & 75,72 & 4,80 & 69,48 & 1,82 \\
\hline Perú & 41,20 & 11,26 & 39,86 & 5,73 & 38,56 & 3,48 \\
\hline Rep. Dominicana & 82,50 & 16,58 & 83,07 & 8,16 & 74,85 & 2,27 \\
\hline Uruguay & 33,47 & 5,67 & 30,50 & 3,36 & 28,64 & 1,77 \\
\hline Promedio Países & 39,92 & 11,64 & 38,31 & 4,71 & 36,96 & 2,40 \\
\hline Total AL & 23,18 & 9,50 & 23,50 & 4,55 & 23,27 & 2,59 \\
\hline
\end{tabular}

Fuente: Elaboración propia 
Cuadro 8. Resultados de proceso de modelamiento multinivel de cuatro niveles para Rendimiento en Matemáticas y Lectura

\begin{tabular}{|c|c|c|c|c|}
\hline & \multicolumn{2}{|c|}{ Rendimiento en Matemáticas } & \multicolumn{2}{|c|}{ Rendimiento en Lectura } \\
\hline & B & (EE) & B & (EE) \\
\hline \multicolumn{5}{|l|}{ Parte fija } \\
\hline Intercepto & 229,56 & $(5,00)$ & 235,20 & $(5,87)$ \\
\hline NSE ${ }^{(1)}$ familia & 1,92 & $(0,27$ & 1,09 & $(0,26)$ \\
\hline Nivel cultural familia & 4,64 & $(0,26)$ & 3,57 & $(0,24)$ \\
\hline Género (varón/mujer) & 2,22 & $(0,41)$ & $-3,96$ & $(0,39)$ \\
\hline Lengua materna (español o portugués /otra) & $-12,57$ & $(1,06)$ & $-7,44$ & $(1,02)$ \\
\hline Años de preescolarización & 1,01 & $(0,14)$ & 1,03 & $(0,14)$ \\
\hline El estudiante tiene computadora & 4,99 & $(0,67)$ & 4,45 & $(0,60)$ \\
\hline Frecuencia de uso de la computadora en la escuela & 1,28 & $(0,23)$ & 0,77 & $(0,22)$ \\
\hline El docente usa la computadora en casa & 3,42 & $(0,97)$ & 2,58 & $(1,11)$ \\
\hline El docente usa la computadora en la escuela & NS & & NS & \\
\hline La escuela tiene más de 10 computadoras & 10,74 & $(1,33)$ & 12,85 & $(3,08)$ \\
\hline \multicolumn{5}{|l|}{ Parte aleatoria } \\
\hline Entre países & 336,89 & & 468,00 & \\
\hline Entre escuelas & 317,49 & & 388,66 & \\
\hline Entre aulas & 96,27 & & 159,96 & \\
\hline Entre alumnos & 1450,10 & & 1300,01 & \\
\hline
\end{tabular}

(1): Nivel socioeconómico.

NS: No significativo a un $\alpha=0,05$.

Fuente: Elaboración propia

Incidencia del acceso y uso de las TIC en el aprendizaje

Tal como se detalla en el apartado metodológico, para analizar la relación entre acceso y uso de TIC y desempeño se siguieron dos procesos de modelado simultáneos, uno para la variable producto Rendimiento en Matemáticas y otro en Lectura.

Del análisis de los Modelos finales, tanto para Matemáticas como para Lectura (cuadro 8), es posible destacar:

1. Los estudiantes que tienen acceso a computadora en casa tienen un logro académico significativamente superior que los que no la tienen. Más concretamente, los datos indican que los estudiantes de sexto grado que tienen acceso a computadoras en casa obtienen 5 puntos en Lectura y de 4,4 en Matemáticas, tras controlarse variables tales como nivel socioeconómico y cultural, género o preescolarización.
2. Existe una relación significativa entre logro escolar y frecuencia de uso de computadora en la escuela. Efectivamente, cuanto más usan la computadora en su escuela los estudiantes obtienen mejores puntuaciones en ambas materias (tras haber controlado el nivel socioeconómico y cultural, el género, la lengua materna y los años de preescolarización). Concretamente, en una escala de 1 (no se utiliza nada) a 5 (se utiliza muy frecuentemente), por cada punto de aumento los estudiantes mejoran su rendimiento 0,8 puntos en Matemáticas y 1,2 en Lectura. De esta forma, las puntuaciones podrían mejorar 3,2 puntos en Matemáticas y 4,8 en Lectura.

3. Los datos señalan que si el docente utiliza la computadora en su casa, el rendimiento de sus alumnos aumenta 3,4 puntos en Lectura y 2,6 en Matemáticas. Sin embargo, si suele utilizar la computadora en la escuela o no, no parece tener efecto en el rendimiento de los estudiantes. 
4. Si la escuela donde están matriculados los niños tiene más de 10 computadoras, el logro académico de los estudiantes será superior, concretamente 12,8 puntos en Matemáticas y 10,7 en Lectura.

Es importante subrayar que los coeficientes de las cuatro variables han hecho una aportación significativa en el Modelo final, es decir, de forma simultánea. Ello implica que sus efectos son aditivos. De esta forma, un estudiante que tenga computadora en casa que utilice frecuentemente (una o dos veces a la semana) la computadora en la escuela, cuyo docente use habitualmente la computadora en su casa y que la escuela donde estudia tenga más de diez computadoras, su resultado será de veintitrés puntos más en Matemáticas y 24,5 puntos más en Lectura. Es decir, casi 0,5 desviaciones típicas, lo que implica un 16,6 por ciento más de puntuación, lo que es del todo impresionante.

\section{Discusión y conclusiones}

El estudio ratifica la importante desventaja con que enfrentan los estudiantes latinoamericanos su proceso educativo. Apenas un tercio de quienes asisten a la educación primaria dispone de una computadora en su hogar, siendo una realidad bastante más compleja para varios de los países de la región $\mathrm{y}$ con fuertes $\mathrm{y}$ graves inequidades entre quienes viven en las zonas rurales o urbanas y el nivel socioeconómico del estudiante. Estos hallazgos son coherentes con los encontrados en otros estudios que abordan la existencia de tecnologías de información y comunicación a nivel de hogares de los estudiantes y, que señalan un significativo mayor acceso entre los estudiantes que habitan zonas urbanas y quienes pertenecen a los sectores sociales de mayores ingresos (SUNKEL, 2007; OCDE, 2010).

Un panorama algo más esperanzador se advierte respecto de la situación a nivel de las escuelas. En efecto, un 82 por ciento de las escuelas a nivel regional, disponen al menos de una computadora. Sin embargo, esta realidad difiere mucho entre países. Por ejemplo, el 84 por ciento de las escuelas en Guatemala y el 71 por ciento de las de República Dominicana, no cuentan con ninguna computadora, mientras que en Chile, Brasil y Cuba, la cobertura es universal.

Por otra parte, las cifras encontradas son bastante menores cuando se trata de computadoras con conectividad o para el uso de los estudiantes y docentes. Al igual que en los casos anteriores, los datos dan cuenta de importantes desigualdades e inequidades entre países y, en su interior según ubicación y nivel socioeconómico de la escuela. Respecto de la disponibilidad de computadoras para el uso de los profesores, es posible configurar grupos de países. Un grupo de avanzada: Brasil, Chile y Cuba en donde la casi totalidad de las escuelas (sobre el 94\%), cuentan con al menos una computadora para el uso de sus docentes. El grupo más rezagado en donde no se supera el 17 por ciento: República Dominicana y Guatemala. Entre medio, países como Argentina, El Salvador y Costa Rica, con porcentajes entre un 44 por ciento y un 54 por ciento. Tales diferencias e inequidades se muestran también en recientes estudios a nivel regional (FALUS; GOLBERG, 2010; SUNKEL, TRUCCO; MÖLLER, 2011).

Más preocupante resulta ser el uso que los estudiantes y docentes señalan hacer de estos recursos en las escuelas. Un 52 por ciento de los estudiantes de $6^{\circ}$ de primaria, afirma que no usan el computador en la escuela, porcentajes que superan el 70 por ciento en Guatemala y Paraguay. Más aún, apenas un 15 por ciento de quienes señalan que la han usado, reconoce un uso frecuente (al menos una vez por semana). Las diferencias por país vuelven a ser lapidarias. Por su parte, mientras un tercio de los profesores declara no usar la computadora en ningún lugar, un magro 36 por ciento dice usarla en las escuelas. La situación por países, permite afırmar que la frecuencia de uso no necesariamente se relaciona con la cantidad disponible de computadoras. Así, hay países con salas de computación en más del 90 por ciento de sus escuelas y en donde 
el porcentaje de docentes que lo usan con frecuencia, no llega a al 45 por ciento. Tal como lo constatan otras investigaciones, el uso de las TIC, no depende exclusivamente de la existencia y disponibilidad de estos recursos en las escuelas.

De cualquier manera, la falta de TIC en los hogares y el bajo uso de las computadoras en las escuelas, está limitando en los estudiantes el acceso y posibilidad de construir conocimiento, al tiempo que los excluye de la comunicación e interacción que emerge y fluye en un mundo global, interconectado y estructurado en redes. Los esfuerzos deben seguir puesto en que ocurra una integración adecuada y eficaz de estos medios tecnológicos, al proceso cotidiano de enseñar y aprender en las escuelas. Disponer de estos recursos, apoyar, incentivar y asegurar el uso entre profesores y estudiantes, es el primer paso para que ello ocurra.

El estudio constata mejores logros cognitivos (en Lectura y Matemáticas), en los estudiantes que disponen de una computadora en su hogar respecto de quienes no cuentan con este recurso en casa. En concreto, su desempeño se ve incrementando en cerca de cinco puntos, una vez que se controla por nivel socioeconómico y cultural de la familia, género o preescolarización del estudiante. Este aporte al logro escolar, es aún más impresionante respecto de la disponibilidad de computadoras en las escuelas. Los estudiantes de $6^{\circ}$ grado que asisten a escuelas con diez o más computadoras, ven incrementado su desempeño en cerca de trece puntos en Matemáticas y once en Lectura. Por su parte, la frecuencia con que los estudiantes usan la computadora en la escuela, se muestra relevante respecto del logro escolar.
Así, mientras más usan la computadora, los estudiantes obtienen mejores logros en Lectura y Matemáticas.

Se encuentra también que los estudiantes cuyos docentes señalan usar la computadora en casa, exhiben un mejor desempeño, en ambas disciplinas, que aquellos cuyos profesores no la usan en casa. Sin embargo, el que los profesores la usen en la escuela, no aparece relacionado con el logro que alcanzan sus estudiantes, una vez que se controla por las variables de ajuste en cuestión. Es muy probable que lo anterior esté relacionado con el tipo de uso dado a la computadora, más que con la frecuencia de uso. Por ejemplo, un uso en casa mayormente enfocado a la preparación de las clases (búsqueda de información, preparación de actividades y materiales, evaluación, otros), mientras que en las escuelas se haga un uso más administrativo y relacional que pedagógico (completar planillas de notas, informes a los padres, etc.). Estos resultados, ameritan seguir indagando y profundizando respecto de la finalidad de uso, tanto como en su incorporación en la práctica pedagógica.

Por último, y dado que todas las variables analizadas han hecho una aportación significativa y simultánea en el modelo final, es posible sostener que: un estudiante que cuente con una computadora en su hogar, que concurra a una escuela con más de diez computadoras, que las utilice al menos una vez por semana y tengan un profesor/a que use habitualmente la computadora en su casa, obtendrá 23 puntos más en Matemáticas y 25 en Lectura. Es decir, casi 0,5 desviaciones típicas, lo que implica cerca de un 17\% más de puntuación que sus pares en la condición inversa.

\section{Referencias}

ANGRIST, Joshua; LAVY, Victor. New evidence on classroom computers and pupil learning. Economic Journal, London, v. 112, n. 482, p. 735-765, 2002.

BENNETT, Sue; MATON, Karl; KERVIN, Lisa. The 'digital natives' debate: a critical review of the evidence. British Journal of Educational Technology, London, v. 39, n. 5, p. 775-786. 2008. 
CARRILLO, Paul; ONOFA, Mercedes; PONCE, Juan. Information technology and student achievement: evidence from a randomized experiment in Ecuador. Washington D.C: BID, 2010.

CARSTENS, Ralph; PELGRUM, Willem (Eds.). Second Information Technology in Education Study (SITES) 2006: technical report. Amsterdam: IEA, 2009.

CHONG, Alberto (Ed). Conexiones del desarrollo: Impacto de las nuevas tecnologías de la información. Serie Desarrollo en las Américas (DIA). Washington D.C: BID, 2011.

CLARO, Magdalena. Impacto de las TIC en Ios aprendizajes de los estudiantes: estado del arte. Santiago de Chile: CEPAL, 2010.

COLL, César. Psicología de la educación y prácticas educativas mediadas por las tecnologías de la información y la comunicación: una mirada constructivista. Sinéctica, Guadalajara, v. 25, p.1-24, 2004.

CONDIE, Rae; et. al. The impact of ict in schools a landscape review. Glasgow: Becta Research, 2007.

DYNARSKI, Mark; et. al. Effectiveness of Reading and Mathematics Software Products: Findings from the First Student Cohort. Washington, D.C.: U.S. Department of Education, Institute of Education Sciences, 2007.

ENGEL, Anna; COLL, César; BUSTOS, Alfonso. Aprender y enseñar con tecnologías de la información y la comunicación en la educación secundaria. In: COLL, César (Coord). Desarrollo, aprendizaje y enseñanza en la educación secundaria. Barcelona: Graó, 2010. p. 105-130

ENG, Ting Seng. The impact of ICT on learning: a review of research. International Education Journal, Armidale, v. 6, n.5, p. 635-650, 2005.

FALUS, Lucía; GOLBER, Mariela. Recursos, instalaciones y servicios básicos en las escuelas primarias de América Latina: otra forma que asume la desigualdad educativa. Cuadernos SITEAL, Buenos Aires, n. 7, p. 01-50. 2010. Disponible en: <http://www. siteal.iipe-oei.org/sites/default/files/siteal_cuaderno07_20101214.pdf>. Acceso en: 24 Julio 2012.

GOOLSBEE, Austan; GURYAN, Jonathan. The impact of internet subsidies in public schools. The Review of Economics and Statistics, Cambridge, v. 88, n. 2, p. 336-347, 2006.

KAZTMAN, R. Impacto social de la incorporación de las nuevas tecnologías de información y comunicación en el sistema educativo. Santiago de Chile: CEPAL, 2010.

KOZMA, Rubén. Monitoring and evaluation of ICT for education impact: a review. In: WAGNER, D.; et. al. Monitoring and evaluation of ICT in education projects: a handbook for developing countries. Washington, D. C.: The World Bank, 2005. p. 11-20.

KULIK, James. Meta-analytic studies of findings on computer-based instruction. In: BAKER, Eva; O'NEIL, Harold. (Eds.). Technology assessment in education and training. Hillsdale, NJ: Lawrence Erlbaum, 1994. p. 09-33.

KULIK, James. Effects of using instructional technology in elementary and secondary schools: what controlled evaluation studies say? Virginia: SRI International, 2003.

LAW, Nancy; PELGRUM, Willem; PLOMP, Tjeerd. (Eds.). Pedagogy and ICT use in schools around the world: findings from the IEA SITES 2006 study. Hong Kong: Springer. Comparative Educational Research Center The University of Honk Kong, 2008.

LAW, Nancy; YUEN, Allan; FOX, Robert. Educational innovations beyond technology: nurturing leadership and establishing learning organizations. New York: Springer, 2011.

MURILLO, Francisco Javier. (Coord.). Investigación Iberoamericana sobre Eficacia Escolar. Bogotá: Convenio Andrés Bello, 2007.

OCDE. ¿Están los Aprendices del nuevo milenio alcanzando el nivel requerido?: uso de la tecnología y resultados educativos en PISA. París: OCDE-ITE, 2010.

PEDRÓ, Francesc. The new millennium learners: challenging our views on ICT and learning. París: OECD-CERI, 2006.

PELGRUM, Willem; LAW, Nancy. ICT en education around de world: trends, problems and prospect. París: IIPE-UNESCO, 2003.

ROMÁN, Marcela. Investigación Latinoamericana sobre Enseñanza Eficaz, ILEE. In: UNESCO. Eficacia escolar y factores asociados en América Latina y el Caribe. Santiago de Chile: UNESCO, 2008, p. 209-225.

ROMÁN, Marcela. Cuatro formas de incorporar las TIC a la enseñanza en el aula. In: BILBAO, A.; SALINAS, Alvaro (Eds.). El libro abierto de la informática educativa: lecciones y desafíos de la red enlaces. Santiago de Chile: Enlaces: Ministerio de Educación, 2010. p. 105-122. 
ROMÁN, Marcela; MURILLO, Francisco Javier. Learning environments with technological resources: a look at their contribution to student performance in Latin American elementary schools. Educational Technology Research and Development, Bloomington, v. 60, n. 6, p. 1107-1128, 2012.

ROSEN, Dina; NELSON, Charles. Web 2.0: a new generation of learners and education. Computers in the Schools, Reno, v. 25, n. 3-4, p. 211-225, 2008.

ROUSE, Cecilia; KRUEGER, Alan. Putting computerized instruction to the test: a randomized evaluation of a 'scientifically based' reading program. Economics of Education Review, Columbia, v. 23, n. 4, p. 323-338, 2004.

SANTIAGO, Ana; et. al. Evaluación experimental del programa "Una laptop por niño" en Perú. Aportes, BID Educación, Washington, D.C., v.5, p.1-12, 2010. Disponible en: <http://idbdocs.iadb.org/wsdocs/getdocument.aspx?docnum=35370099>. Acceso en mayo 2012.

SELWYN, Neil. Reconsidering political and popular understandings of the digital divide. New Media \& Society, v. 6, n. 3, p. 341-362, 2004.

SELWYN, Neil. Schools and schooling in the digital age: a critical perspective. London: Routledge, 2011.

SUNKEL, Guillermo. Las tecnologías de la información y la comunicación (TIC) en la educación en América Latina: una exploración de indicadores. Santiago de Chile: CEPAL, 2007.

SUNKEL, Guillermo; TRUCCO, Daniela; MÖLLER, Sebastián. Aprender y enseñar con las tecnologías de la información y las comunicaciones en América Latina: potenciales beneficios. Santiago: CEPAL, 2011.

TRUCANO, Michael. Knowledge maps: ICT in education. Washington, DC: The World Bank, 2005.

UNESCO. Los aprendizajes de los estudiantes de América Latina y el Caribe: primer reporte SERCE. Santiago de Chile: LLECEOREALC/UNESCO, 2008.

VALIENTE, Oscar. 1-1 in education: current practice, international comparative research evidence and policy implications. OECD Education Working Papers, Paris, n. 44, p. 01-20, 2010.

VENEZKY, Richard. Quo vademus?: the transformation of schooling in a networked world. París: OCDE/CERI, 2002.

WEBB, Mary; COX, Margaret. A review of pedagogy related to ICT technology. Pedagogy and Education, Luton, v. 13, n. 3, p. 235-286, 2004

Recebido en: 23.07.2013

Aprobado en:16.10.2013

Marcela Román Investigadora principal del Centro de Investigación y Desarrollo de la Educación (CIDE) y académica de la Facultad de Educación de la Universidad Alberto Hurtado, Chile. Investigadora asociada al Centro de Prácticas y Políticas Educativas, CEPPE, de la Pontificia Universidad Católica de Chile. Directora de la Revista Iberoamericana de Evaluación Educativa.

F. Javier Murillo Profesor Titular de Universidad en Métodos de Investigación y Evaluación en Educación, Universidad Autónoma de Madrid. Director del Doctorado en Educación de la UAM, y coordinador de la Red Iberoamericana de Investigación sobre Cambio y Eficacia Escolar (RINACE). 